\title{
Hohlfaserbasiertes Lab-on-a-Chip Dual-Perfusions-System mit integrierter fluoreszenzbasierter Sauerstoffüberwachung
}

\author{
C. Winkelmann ${ }^{1}$, F. Schmieder ${ }^{1}$, S. Grünzner ${ }^{1}$, U. Marx ${ }^{2}$, F. Sonntag ${ }^{1}$ \\ ${ }^{1}$ Fraunhofer-Institut für Werkstoff- und Strahltechnik IWS, Dresden, Deutschland \\ ${ }^{2}$ Technische Universität Berlin, Institut für Biotechnologie, Berlin, Deutschland
}

\begin{abstract}
Abstrakt:
Entwicklungen zur Generierung komplexer 3D-Gewebekulturen gewinnen für die pharmazeutische und biochemische Industrie stetig an Bedeutung. Dabei bestehen die Herausforderungen in der Aufrechterhaltung des biologischen 3D-Mikromilieus bei gleichzeitiger Gewährleistung möglicher Interaktionen verschiedener Zelltypen oder Gewebe innerhalb eines mikrofluidischen Systems. Ein Lösungsansatz bietet die Einbindung eines Hohlfasernetzwerks, das als künstliches Gefäßsystem fungiert. Versorgt wird dieses über ein angeschlossenes mikrofluidisches Perfusions-System, das über integrierte Mikropumpen betrieben wird. Dieser als Lab-on-a-Chip-System ausgelegte Bioreaktor ermöglicht durch die Einbindung mehrerer separat angeordneter mikrofluidischer Kreislaufsysteme einen dualen Perfusionsbetrieb. Zur Charakterisierung der Eigenschaften und zur Überwachung der Zellkultur ist die Adaption eines applikationsspezifischen, ortsaufgelösten Online Monitoring Systems notwendig. Dazu wird ein fluoreszenzbasierten Messsystem vorgestellt, das eine integrierte Überwachung des Sauerstoffgehalts während der Kultivierung ermöglicht.
\end{abstract}

Schlüsselwörter: Bioreaktor, Lab-on-a-Chip, Perfusionssystem, Sauerstoffüberwachung, Fluoreszenzmessung

\section{Einleitung}

Die in vitro Substanztestung erfordert die Bewertung von Interaktionen verschiedener Zelltypen oder Gewebe in einem geschlossenen Kreislaufsystem [1, 2]. Daher ist es unser Ziel, ein dynamisches Mikro-Bioreaktorsystem mit einem integrierten, künstlichen Blutgefäßsystem zu entwickeln. Es soll ein komplexes, biokompatibles dreidimensionales Mikromilieu geschaffen werden. Eine Möglichkeit zur Realisierung eines biofunktionalen, vaskularisierten Systems besteht in der Verwendung von Hohlfasern [3]. Diese müssen mit der Mikrofluidik gekoppelt werden, um die Versorgung des künstlichen Gewebes mit Sauerstoff und Nährstoffen gewährleisten zu können. Die größte Herausforderung besteht in der biokompatiblen und fluidisch dichten Verbindung beider Komponenten [4]. Die Charakterisierung der fluidischen Eigenschaften erfolgt mittels Particle Image Velocimetry (PIV), wohingegen die Permeabilität mittels Fluoreszenz- und radiologischen Kontrastmitteluntersuchungen bestimmt wurde.

\section{Material und Methode}

\subsection{Hohlfaserbasiertes Lab-on-a-Chip Dual- Perfusions-System}

Bei dem hohlfaserbasierten Lab-on-a-ChipSystem handelt es sich um einen dualen Perfusions-Mikro-Bioreaktor (siehe Bild 1).

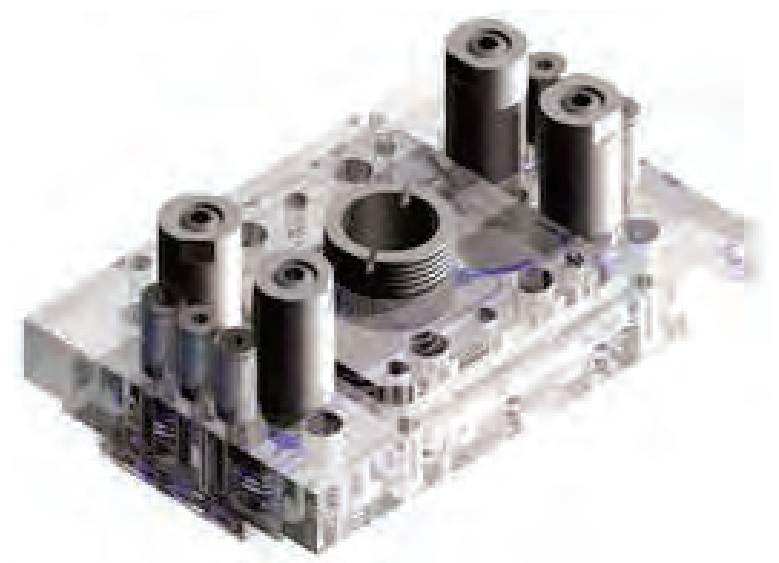

Bild 1: Schematische Darstellung des hohlfaserbasierten Lab-on-a-Chip Dual-Perfusions-Systems. 
Das System basiert auf der etablierten Multi-Organ-Chip (MOC)-Plattform, die in Kooperation zwischen dem Fraunhofer IWS und dem Institut für Biotechnologie der Technischen Universität Berlin entwickelt wurde [1, 2, 5, 6].

Es umfasst mehrere integrierte Zellkulturräume, Medienreservoire, Mikroventile und -pumpen, die eine gleichzeitige Kultivierung unterschiedlicher Gewebearten ermöglichen. Durch ein geschlossenes fluidisches Kreislaufsystem im Mikrometerbereich, über das die einzelnen Zellkulturräume untereinander gekoppelt sind, können die Zellen miteinander interagieren [4]. Der Medienfluss wird über integrierte, pneumatisch betriebene Mikropumpen realisiert, deren Antrieb über ein automatisiertes Steuermodul erfolgt. Gegenwärtig kommen für die Kultivierung in den Zellkulturräumen austauschbare Zellkultureinsätze zur Anwendung.

Darauf basierend wurde ein neuartiges Lab-ona-Chip-System entworfen, das die Kultivierung komplexer Zell- und Gewebekulturen über zwei separat angelegte Perfusionskreisläufe realisiert (siehe Bild 2).

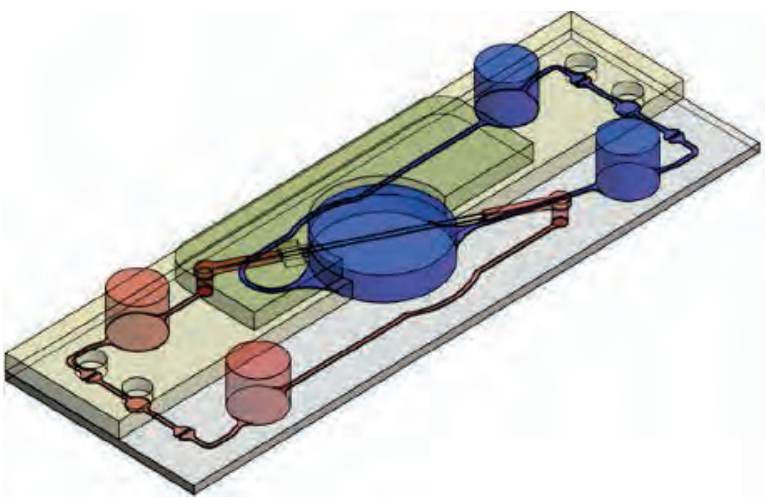

Bild 2: Mikrofluidikdesign der Flusszellen des Lab-ona-Chip Dual-Perfusions-Bioreaktors mit zwei separat angelegten Perfusionskreisläufen (rot und blau).

Das modulare Konzept des Perfusionssystems bietet die Möglichkeit der individuellen Gestaltung und applikationsspezifischen Designanpassung der mikrofluidischen Kanalstruktur, ohne komplette konstruktive und fertigungstechnische Neugestaltung des Bioreaktorsystems vornehmen zu müssen. Beispielsweise kann durch konzeptionelle Auslegung der Medienfluss in Kreuz- oder Gegenstromrichtung realisiert werden.

Eine Möglichkeit zur Kopplung der beiden Kreislaufsysteme bietet die Einbindung von permeablen künstlichen Hohlfasern. Über diese und die unterschiedlichen Medienkreisläufe können verschiedene Zellen mit Nährstoffen und Sauerstoff versorgt werden und gleichzeitig untereinander interagieren. Die Durchströmung wird über integrierte Mikroventile und -pumpen umgesetzt und über ein automatisiertes Steuermodul individuell angepasst.

Für die Integration der Hohlfasern wurde das Lab-on-a-Chip-System entsprechend dem neuen Anforderungsprofil angepasst (siehe Bild 3).

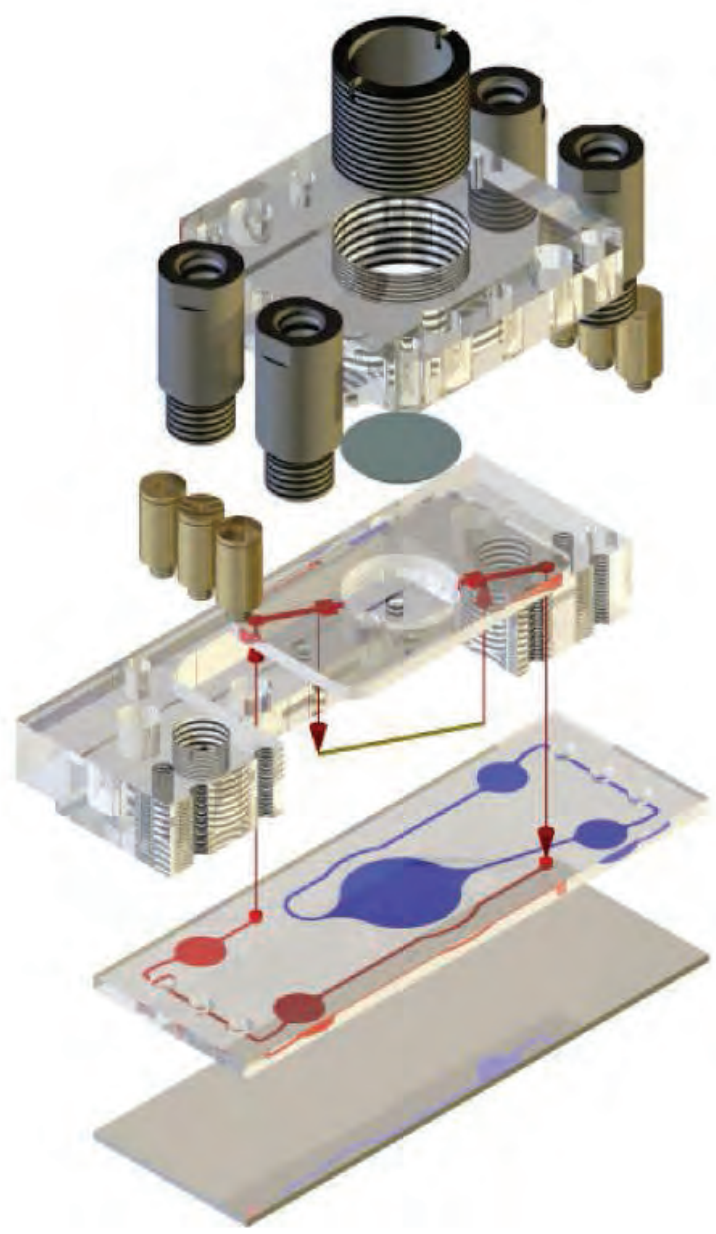

Bild 3: Aufbau des, mit einem Hohlfasereinsatz konfigurierten, Lab-on-a-Chip Dual-PerfusionsSystems.

\subsection{Artifizieller Verbraucher auf Enzymbasis}

Auf Grund der schwierigen Reproduzierbarkeit von Zellkulturexperimenten sind deren Ergebnisse nur bedingt vergleichbar. Zudem erfordern die Versuche einen hohen materiellen und zeitlichen Aufwand. Aus diesem Grund wurde ein einfacheres Verbrauchermodell für Vorversuche entwickelt. Dabei wird anstelle von Zellen das Enzym Glucoseoxidase, mit dem ein Sauerstoffverbraucher simuliert wird, verwendet.

Die induzierte, enzymkatalysierte Reaktion verläuft dabei nach dem folgenden Schema: 


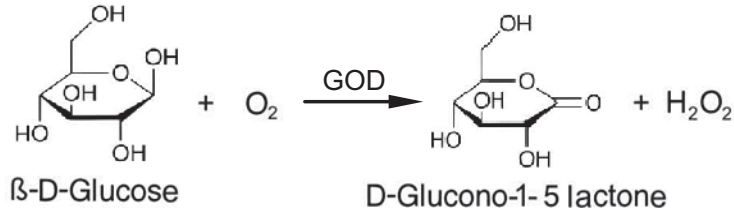

Bild 4: Glucoseoxidase-Reaktion (GOD = Glucoseoxidase).

Um individuell den Sauerstoffverbrauch einer Zellkultur im Dual-Perfusions-Bioreaktor simulieren zu können, muss das Enzym ortsselektiv und mengenspezifisch in der Zellkulturkammer immobilisiert werden. Die Versorgung erfolgt über das integrierte Hohlfasernetzwerk des Perfusions-Mikro-Bioreaktorsystems. Auf diese Weise kann die erforderliche Auslegung der Hohlfasern, deren Anzahl und Anordnung sowie entsprechend spezifische Pumpenparameter definiert werden, um eine ausreichende Versorgung gewährleisten zu können.

\subsection{Sauerstoffsensitive Mikropartikel zur fluoreszenzbasierten Sauerstoffmessung}

Bei optischen Sauerstoffmessungen werden Farbstoffe verwendet, deren Fluoreszenzverhalten sich in Abhängigkeit vom Sauerstoffgehalt ändert. Beispielsweise eignen sich für die Anwendung Ruthenium-Phenanthrolin- und Platin-Porphyrin-Derivate [7].

Liegt molekularer Sauerstoff $\left(\mathrm{O}_{2}\right)$ vor, wird die Fluoreszenz dynamisch gelöscht (Quenching). Bei Kontakt überträgt der Farbstoff Energie spezifisch auf das Sauerstoffmolekül. Dadurch nehmen die Intensität der Fluoreszenz und die Abklingzeit mit zunehmender Sauerstoffkonzentration ab [7].

Für die Online-Überwachung des Sauerstoffgehalts werden CPOx-Beads der Firma Colibri Photonics angewendet. Bei diesen fluoreszierenden, sauerstoffsensitiven Mikropartikeln ist der Fluoreszenzfarbstoff von einer sauerstoffdurchlässigen Polymerhülle umgeben, die gleichzeitig die Intensität der Fluoreszenz steigert. Substanzen, wie $\mathrm{CO}_{2}, \mathrm{~N}_{2}, \mathrm{CH}_{4}$, Wasser, $\mathrm{H}^{+}$, weitere Ionen, Inhaltsstoffe von Nährmedien (Zucker, Eiweiß etc.) beeinflussen den Fluoreszenzprozess nicht [7].

Die Integration der CPOx-Beads in das Fluidiksystem des hohlfaserbasierten Lab-on-a-Chip Dual-Perfusions-System stellt die Basis für eine ortsselektive fluoreszenzbasierte Überwachung des Sauerstoffgehalts während der Zellkultivierung dar [7].

\subsection{Fluoreszenzlebensdauerbasierte Sauer- stoffüberwachung}

Um den Eintrag und den Verbrauch von Sauerstoff in chemischen, biochemischen und biologischen Prozessen zu ermitteln, wurde auf Grundlage des OPAL-Systems von Colibri Photonics ein System zur nichtinvasiven Messung der Sauerstoffkonzentration entwickelt.

Dafür wurden Software und Elektronik des OPAL-Systems mit dem am Fraunhofer IWS entwickelten, frei konfigurierbaren Fluoreszenzmessmodul [8] kombiniert. Das Fluoreszenzmodul wurde den Farbstoffen entsprechend mit einer geeigneten Diode (520 nm Wellenlänge) und passenden Filtern ausgestattet (siehe Bild 5).

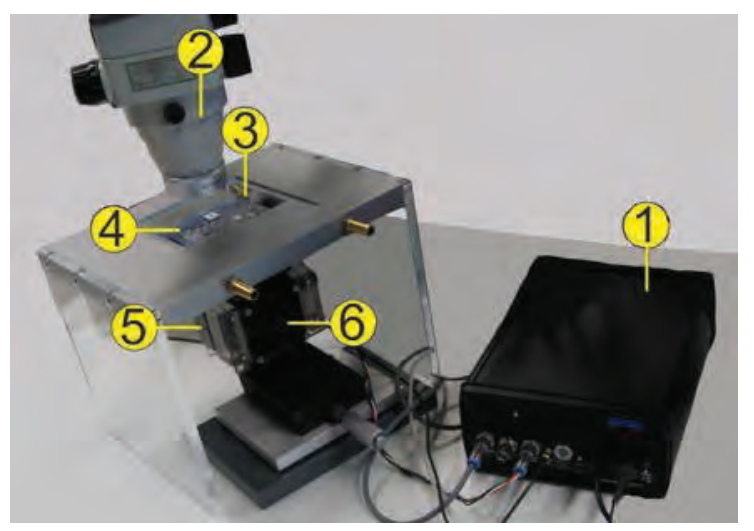

\begin{tabular}{|c|l|}
\hline 1 & Elektronik des OPAL-Systems \\
\hline 2 & $\begin{array}{l}\text { Auflichtmikroskop zur optischen } \\
\text { Kontrolle der Position der } \\
\text { Fluoreszenzbeads }\end{array}$ \\
\hline 3 & $\begin{array}{l}\text { Temperatursensor Pt 100 zur } \\
\text { Temperaturkompensation während der } \\
\text { Sauerstoffmessung }\end{array}$ \\
\hline 4 & Perfusions-Mikro-Bioreaktorsystem \\
\hline 5 & $\begin{array}{l}\text { für Sauerstoffmessung konfiguriertes } \\
\text { Fluoreszenzmodul }\end{array}$ \\
\hline 6 & $\begin{array}{l}\text { manuell verstellbare Z-Achse zur } \\
\text { Einstellung der Fokuslage }\end{array}$ \\
\hline
\end{tabular}

Bild 5: Modifiziertes Fluoreszenzmesssystems zur fluoreszenzlebensdauerbasierte Sauerstoffmessung.

Die Lichtleistung des Messsystems ist bis zu $1 \mathrm{~W}$ regelbar. Die Ortsauflösung beträgt bis $z u$ $500 \mu \mathrm{m}$. Entsprechend den Anforderungen der Anwendung kann die Messdauer von $1 \mathrm{~s}$ bis zu einem kontinuierlichen Betrieb individuell eingestellt werden. Mehrfachmessungen sind über die Vorgabe definierter Zeitintervalle möglich.

Durch die geringe Größe des Messkopfes von nur $90 \times 100 \times 25 \mathrm{~mm}^{3}$ ist das System flexibel als Messeinheit an einem Portalroboter oder als „Stand-Alone-Modul“ einsetzbar. 
Über die Kopplung an ein Portalrobotiksystem ist die automatisierte, ortsaufgelöste Messung des Sauerstoffgehaltes möglich.

\section{Ergebnisse}

\subsection{Prototyp des hohlfaserbasierten Lab-on- a-Chip Dual-Perfusions-System}

Entsprechend der Konzeptentwicklung konnten erfolgreich erste Modelle des hohlfaserbasierten Lab-on-a-Chip Dual-PerfusionsSystems realisiert werden (siehe Bild 6).

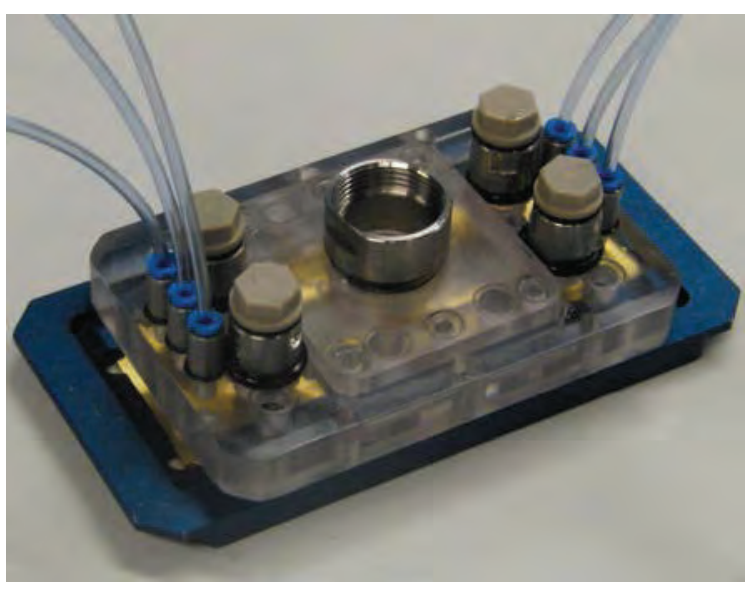

Bild 6: Applikationsspezifisch modifizierter Lab-on-aChip Dual-Perfusions-Bioreaktor mit zwei separat angelegten Perfusionskreisläufen und integrierter Hohlfaser.

Die Mikrokanalstruktur dieses Systems umfasst eine Breite von 500 bis $750 \mu \mathrm{m}$ und ist 100 bis $160 \mu \mathrm{m}$ hoch.

Durch die Verarbeitung von biokompatiblen, transparenten Materialien wurde der DualPerfusions-Bioreaktor auf die Überwachung mit optischen online-monitoring Systemen ausgelegt.

\subsection{Integration der Hohlfaser in das hohlfaserbasierte Lab-on-a-Chip Dual- Perfusions-System}

Die künstliche Hohlfaser konnte erfolgreich in das Dual-Perfusions-System integriert werden. Es erfolgte zunächst die Einbettung einer einzelnen Hohlfaser in die Kanalstruktur (siehe Bild 7).

Für die ersten Versuche wurde eine mikroporöse kunststoffbasierte Hohlfaser (MicroPES ${ }^{\circledR}$ Capillary Membrane der Firma Membrana $\mathrm{GmbH}$ ) in das System integriert.
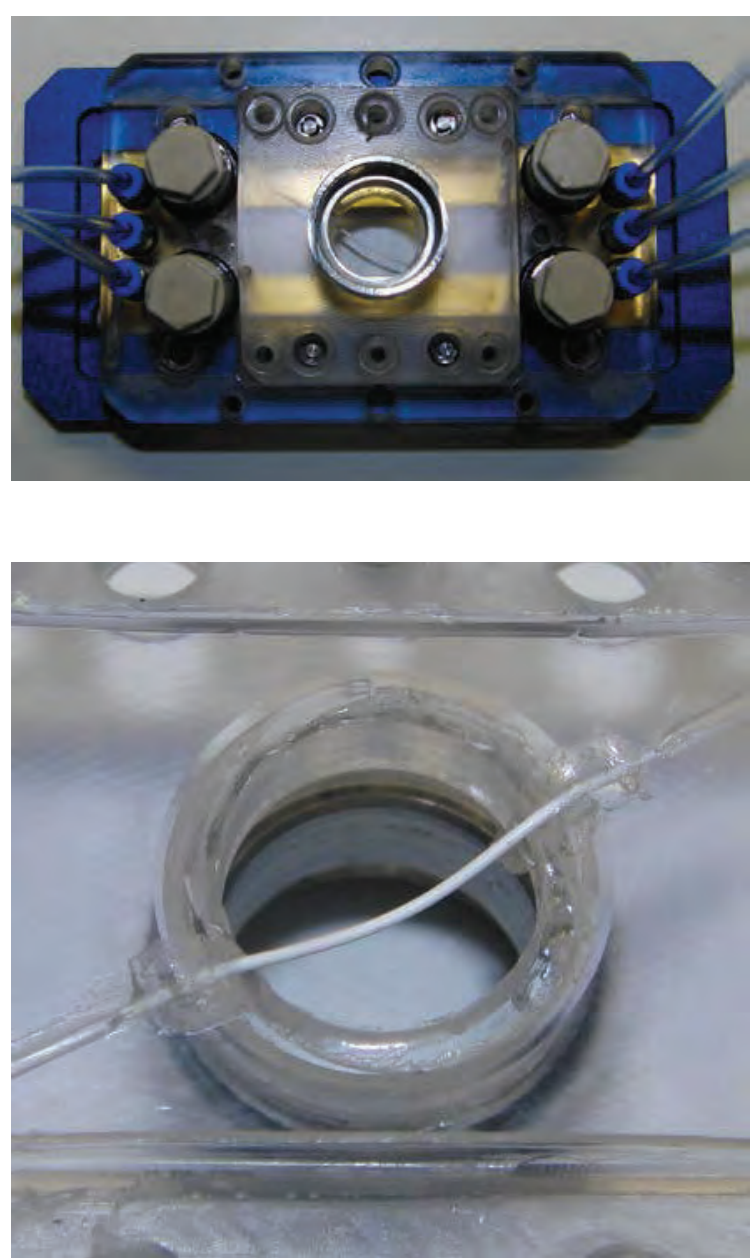

Bild 7: Integrierte mikroporöse, kunststoffbasierte Hohlfaser im Dual-Perfusions-Bioreaktor.

Unter Anwendung eines mit Kieselsäure angereicherten Polydimethylsiloxans (PDMS, Sylgard®184, Dow Corning) konnte eine fluidisch dichte Verbindung der Trennebenen erfolgreich realisiert werden.

\subsection{Immobilisierung von sauerstoffsensi- tiven, fluoreszenzbasierten CPOx-Beads in das Lab-on-a-Chip Dual-Perfusions-System}

Für die ortsaufgelöste fluoreszenzlebensdauerbasierte Sauerstoff-Überwachung müssen sich die Beads auf dem Glasboden der Zellkulturkammer des Dual-Perfusions-Bioreaktors lokal immobilisieren lassen (siehe Bild 8). 


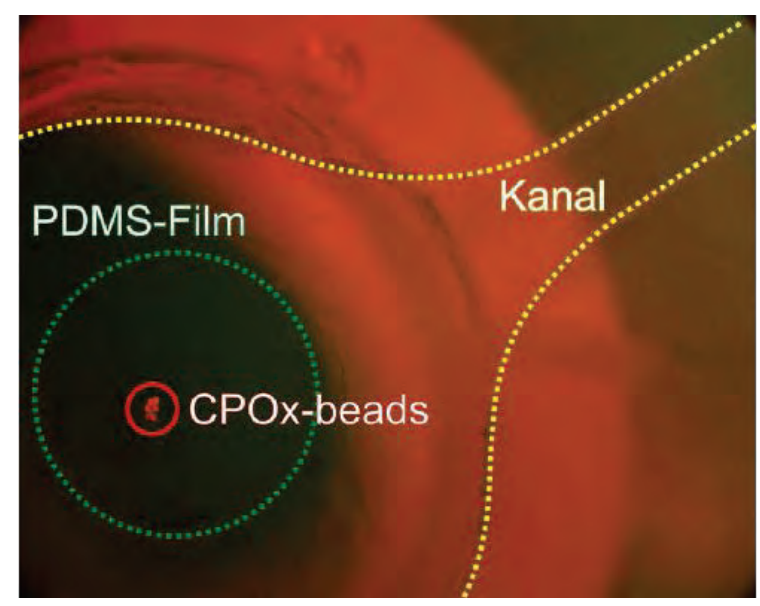

Bild 8: Immobilisierte, sauerstoffsensitive Fluoreszenzbeads im Zellkulturraum des Dual-PerfusionsBioreaktors.

Dazu wurden erfolgreich Versuche mit verschieden Silikonen durchgeführt (siehe Tab. 1).

Tab. 1: Materialien zur Immobilisierung von CPOxBeads

\begin{tabular}{|c|c|}
\hline Silikonart & $\begin{array}{c}\text { Ergebnis der } \\
\text { Immobilisierung }\end{array}$ \\
\hline PDMS DC Sylgard 184 & $\checkmark$ \\
\hline Klebesilikon DC 9600 & $\checkmark$ \\
\hline Klebesilikon DC 9800 & \\
\hline
\end{tabular}

\subsection{Referenzierung und Kalibrierung des Fluoreszenzmoduls zur online- Überwachung des Sauerstoffverbrauchs}

Die Nullreferenzierung des Systems erfolgte mittels einer wässrigen Rodamin B-Lösung mit $\mathrm{C}_{\text {Rhodamin } \mathrm{B}}=10^{-2} \mathrm{~mol} / \mathrm{L}$.

Die Kalibration für einen Sauerstoffgehalt von $100 \%$ wurde auf Basis einer Suspension aus demineralisiertem Wasser und FluoreszenzBeads durchgeführt. Für die $0 \%$ Kalibration wurde das demineralisierte Wasser mit Stickstoff begast. Dabei trat bei wiederholten Messungen eine Abweichung der Mittelwerte von ca. $2 \%$ an unterschiedlichen Tagen auf. Daher ist eine Kalibration des Systems mindestens einmal täglich notwendig.

\subsection{Fluoreszenzlebensdauerbasierte Sauer- stoffüberwachung auf Basis des enzymatischen Verbrauchers}

Um das fluoreszenzlebensdauerbasierte Sauerstoffmesssystem zu charakterisieren, kam die enzymkatalysierte, sauerstoffverbrauchende Reaktion von Glucose zu Glucono-1,5-lacton als artifizieller Verbraucher zur Anwendung. Dazu wurde in einem Probesystem eine hochkonzentrierte Glucose-Lösung vorgelegt und im Anschluss eine hochkonzentrierte Glucoseoxidase-PBS-Lösung hinzu pipettiert. Durch den Überschuss von Glucose und Enzym konnte so die sauerstofflimitierende Reaktion und damit die Sauerstoffmessung charakterisiert werden (siehe Bild 9).

\section{2-Gehalt in der Lösung in \%}

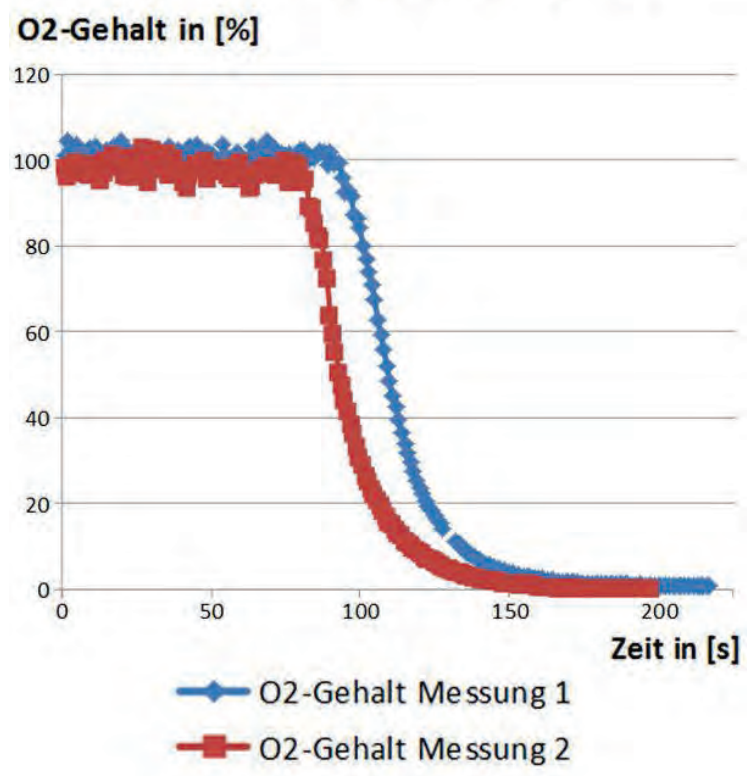

Bild 9: Darstellung der gemessenen Sauerstoffkonzentration im Medium während der enzymkatalysierten Reaktion.

Es wurde erfolgreich nachgewiesen, dass sich diese enzymatische Reaktion zur Simulation eines Sauerstoffverbrauchers eignet. Im Vergleich zur Zellkultur lässt sich diese besser steuern und schafft bei Mehrfach-Versuchen besser reproduzierbare Bedingungen. Dieses Modell liefert daher vergleichbare Ergebnisse, um Aussagen zu geeigneten Hohlfaserkonfigurationen treffen zu können.

\section{Zusammenfassungen}

Für die Ausbildung und Aufrechterhaltung von biologischen 3D-Mikromilieus wurde erfolgreich ein Lab-on-a-Chip Dual-Perfusions-System entwickelt und etabliert.

In dieses System konnten erfolgreich mikroporöse kunststoffbasierte Hohlfasern, die als künstliches Gefäßsystem fungieren, fluidisch dicht integriert und über ein Mikropumpensystem perfundiert werden.

Für die Charakterisierung wurde erfolgreich ein zellfreies Modell zur Simulation eines Sauerstoffverbrauchers entwickelt, das auf der enzymatischen und sauerstoffverbrauchenden Glucoseoxidase-Reaktion basiert. Im Vergleich 
zu Zellkulturexperimenten werden reproduzierbarere Bedingungen geschaffen, wodurch vergleichbare Ergebnisse geliefert werden, um Aussagen zu geeigneten Hohlfaserkonfigurationen treffen zu können.

Zur Beurteilung der Funktionalität des Lab-ona-Chip Dual-Perfusions-Systems konnte erfolgreich ein optisches Sauerstoffmesssystem als neuartige Technologie zur ortsaufgelösten Überwachung des Sauerstoffgehaltes von Zellkulturen entwickelt und etabliert werden.

Die für eine Messung erforderlichen sauerstoffsensitiven Fluoreszenzbeads CPOx lassen sich mittels Silikonen wie PDMS DC Sylgard 184, DC 9600 und DC 9800 im Lab-on-a-Chip DualPerfusions-Systems erfolgreich immobilisieren.

Die prinzipielle Funktionalität des fluoreszenzbasierten Sauerstoffmesssystems konnte über die Simulation des Sauerstoffverbrauchs auf Basis der Glucoseoxidase-Reaktion erfolgreich nachgewiesen werden.

\section{Ausblick}

Mittels der fluoreszenzlebensdauerbasierten Sauerstoffmesstechnologie sind Untersuchungen zur Charakterisierung und Optimierung des hohlfaserbasierten Lab-on-a-Chip DualPerfusions-Systems geplant.

Dieser Ansatz ermöglicht die Auslegung bzw. Dimensionierung des erforderlichen Hohlfasernetzwerks zur Gewährleistung einer ausreichenden Sauerstoffversorgung.

Durch die Charakterisierung der fluidischen Eigenschaften soll die Optimierung des Mikrofluidiksystems erfolgen.

Dazu sind weitere Untersuchungen zur Bestimmung der technologischen Grenzen im Hinblick auf die Permeabilität, Durchströmbarkeit und Dichtigkeit des Systems in Abhängigkeit der Variation von Prozessparametern der Pumpensteuerung geplant.

\section{Danksagung}

Die Autoren danken dem Freistaat Sachsen und der Europäischen Union (SAB Projekt UNILOC), dem Europäischen Sozialfond und dem SMWK (Stipendium Claudia Winkelmann) sowie dem BMWI (ZIM Projekt RoVitaS) für die finanzielle Unterstützung.

\section{References}

[1] U. Marx, H. Walles, S. Hoffmann, G. Lindner, R. Horland, F. Sonntag, U. Klotzbach, D. Sakharov, A. Tonevitsky und R. Lauster: 'Human-on-a-chip' Developments: A Translational Cuttingedge Alternative to Systemic Safety Assessment and Efficiency Evaluation of Substances in Laboratory Animals and Man? In: at - ATLA (2012) Nr. 40, S. 235-257.

[2] U. Marx, B. Atac, S. Brincker, M. Busek, C. Drewell, R. Eger, M. Fischer, C. Frädrich, D. Geßner, T. Hasenberg, S. Hoffmann, R. Horland, A. Jaenicke, L. Kuba, R. Lauster, G. Lindner, A. Lorenz, E.-M. Materne, M. Rosowski, K. Schimek, U. Süßbier, I. Wagner, L. Kloke und U. Menzel: Developing a human chip-based platform for repeated dose toxicity testing. In: at - AXLR8 Alternative Testing Strategies Progress Report (2012), S. 210-218.

[3] W. Meyer, S. Engelhardt, E. Novosel, B. Elling, M. Wegener, H. Krüger: Soft Polymers for Building up Small and Smallest Blood Supplying Systems by Stereolithography. In: at - Journal of Functional Biomaterials (2012) Nr. 3, S. 257-268

[4] F. Sonntag, M. Gruchow, I. Wagner, G. Lindne und U. Marx: Miniaturisierte humane organtypische Zell- und Gewebekulturen. In: at BIOspektrum (2011) Nr. 4, S. 418-421.

[5] F. Sonntag, M. Gruchow, I. Wagner, G. Lindner und U. Marx: Miniaturisierte humane organtypische Zell- und Gewebekulturen. In: at BIOspektrum (2011) Nr. 17, S. 418-421.

[6] C. Winkelmann; Y.Luo, A. Lode, M. Gelinsky, U. Marx, M. Busek, F. Schmieder, F. Sonntag: Charakterisierung von in Lab-on-a-Chip Systemen eingebetteten Hohlfasern; tm Technisches Messen: Vol. 80, No. 5 (2013), S. 147-154.

[7] E. Schmälzlin, J. van Dongen, I. Klimant, B. Marmodée, M. Steup, J. Fisahn, P. Geigenberger, H.-G. Löhmannsröben: An optical multi-frequency phase modulation method using micro-beads for measuring intracellular oxygen concentrations in plants; Biophys. J. 89 (2005) 1339-1345.

[8] F. Schmieder, R. Eger, S. Schmieder, M. Ruhnow, S. Friedrich, N. Danz, U. Marx und F. Sonntag: Automated universal chip platform for fluorescence based cellular assays. In: Biomed Tech 2012; 57 (Suppl. 1), DOI: 10.1515/bmt2012-4319. 